\title{
Intérêt des techniques de préservation d'une baguette osseuse mandibulaire associée à une reconstruction par lambeau libre de fibula dans les réhabilitations buccales
}

\author{
Phulpin $\mathbf{B}^{1,2}$, Cortese $S^{2}$, Gallet $\mathbf{P}^{2}$, Mastronicola $\mathbf{R}^{2}$, Gangloff $\mathbf{P}^{1,2}$, Bravetti $\mathbf{P}^{3,}$ \\ Verhaeghe $\mathrm{JL}^{4}$, Dolivet $\mathrm{G}^{2}$ \\ ${ }^{1}$ Service d'Odontologie, Centre Alexis Vautrin, Vandoeuvre les Nancy, France \\ ${ }^{2}$ Unité de Chirurgie cervico-faciale, Centre Alexis Vautrin, Vandoeuvre les Nancy, France \\ ${ }^{3}$ Service de Chirurgie buccale, Faculté d'Odontologie, Nancy, France \\ ${ }^{4}$ Département de Chirurgie oncologique, Centre Alexis Vautrin, Vandoeuvre les Nancy, France \\ b.phulpin@nancy.fnclcc.fr
}

Contexte: Les interventions chirurgicales comportant une résection de la région antérieure de la mandibule nécessitent une reconstruction immédiate afin de restaurer l'esthétique et la fonction. Elle est le plus souvent réalisée avec une greffe microvascularisée de fibula. Cette technique comporte des difficultés sur le plan esthétique pour le rétablissement du contour mandibulaire, et sur le plan fonctionnel si les articulations temporo-mandibulaires n'ont pas été correctement réalignées. Pour la réhabilitation buccale, la qualité et le volume de la fibula permettent la mise en place d'implants dentaire (Hayter et Cawood 1996, Frodel et al. 1993). Cette technique comporte un facteur limitant: le faible diamètre dyaphysaire de la fibula $(15 \mathrm{~mm})$, comparé à la hauteur l'os natif mandibulaire, ne permet pas de restaurer simultanément l'os basal et l'os alvéolaire. Par conséquent, il existe un différentiel osseux entre l'os résiduel et le greffon, particulièrement chez les patients dentés, qui rend difficile le rétablissement du plan d'occlusion (Iriarte et Reychler 1993). Enfin, la réhabilitation prothétique nécessite la mise en place de suprastructures très longues, entraînant un risque de fracture et de mauvais résultats esthétiques.

Objectif: L'objectif de cette technique chirurgicale qui, en préservant une baguette osseuse mandibulaire, est de restaurer la hauteur mandibulaire et de rétablir facilement l'articulé dentaire avec un lambeau libre de fibula.

Patients et méthodes : quatre patients (4 hommes, âge moyen 61 ans) ont été traitées par cette méthode. Il s'agissait dans 3 cas d'un traitement carcinologique et dans un cas de la prise en charge d'une ostéoradionécrose. Lors de l'intervention chirurgicale, une section horizontale parallèle au bord basilaire de la mandibule, permettant la conservation d'une baguette mandibulaire de 3 à 5 $\mathrm{mm}$, a été réalisée. Puis, des ostéotomies verticales et parallèles rejoignant la section horizontalement sont effectuées. L'exérèse en bloc est alors réalisée et un lambeau infra-hyoïdien est positionné pour restaurer le plancher de bouche. Le greffon fibulaire est ensuite transféré, puis stabilisé sur le site receveur. Une évaluation carcinologique, fonctionnelle et esthétique a été réalisée.

Résultats : Un patient est décédé au $12^{\text {ème }}$ jour postopératoire, d'une affection indépendante de l'intervention. Pour les 3 autres patients, les résultats esthétiques et fonctionnels se sont avérés satisfaisante, avec une bonne restauration de la hauteur de l'os mandibulaire. D'un point de vue carcinologique, aucune récidive locale n'a été constatée.

Conclusion: Cette technique donne une restauration mandibulaire esthétique et fonctionnelle satisfaisante. Ces patients sont actuellement en cours d'analyse pré-implantaire. Le volume et la qualité du tissu osseux disponible permettra la mise en place sans difficultés d'implants dentaires, avec une conception prothétique ultérieure simplifiée en raison de la préservation de la hauteur mandibulaire. 